\title{
Estudio comparativo en rendimiento y calidad de 12 variedades de quinua orgánica en la comunidad campesina de San Antonio de Manallasac, Ayacucho
}

Comparative study in performance and quality of 12 organic quinua varieties in the peasant community of San Antonio de Manallasacc,

Ayacucho

Recibido: setiembre 02 de 2019 | Revisado: noviembre 05 de 2019 | Aceptado: diciembre 13 de 2019

\author{
Máximo Soto Pardo ${ }^{\mathrm{I}}$ \\ Rolando Allende Burga ${ }^{\mathrm{I}}$ \\ Violeta L. Romero CARrión ${ }^{2}$
}

\begin{abstract}
Resumen
Diferentes variedades de quinua procedentes de distintas zonas fueron adaptadas en la comunidad campesina San Antonio de Manallasacc del distrito de Chiara, provincia de Huamanga y Región de Ayacucho - Perú ubicada a 3520 metros sobre nivel del mar para observar su comportamiento en cuanto a rendimiento y calidad en condiciones distintas a su origen. El experimento se realizó en parcelas ubicadas en el campo y en condiciones climatológicas naturales. Las variedades de quinua utilizadas fueron Amarilla Marangani, Illpa Inía, Inía Salcedo, Altiplano, Hualhuas, Rosada Junín, Huancayo, Inía 433, Pasankalla, Negra Collana, Amarilla Sacaca y Blanca Junín. Las condiciones agroclimáticas así como el suelo y el agua resultaron favorables para la producción de quinua. Los resultados obtenidos fueron los siguientes: la variedad INIA 433 logró el mayor rendimiento con $4.72 \mathrm{Tm}$ por hectárea y $14.3 \%$ de proteína. Es una planta de panoja grande y uniforme. También se observó homogeneidad de las plantas en el crecimiento y desarrollo. La variedad de mayor proteína fue la Amarilla Marangani con 16\% de proteína. Las variedades de quinua precoces fueron Illpa Inía, Inía Salcedo, Altiplano y Pasankalla con (150-160 días de período vegetativo).
\end{abstract}

Palabras clave: Ayacucho, quinua, variedades, calidad, rendimiento
1 Facultad de Ingeniería Industrial y de Sistemas, Universidad Nacional Federico Villarreal, Lima, Perú.

2 Escuela Universitaria de Posgrado, Universidad Nacional Federico Villarreal, Lima, Perú. vromero@unfv.edu.pe

\begin{abstract}
Different quinoa varieties from other areas were adapted in the peasant community of San Antonio de Manallasacc, of Chiara district, Province of Huamanga in Ayacucho - Peru, located at 3520 meters above sea level to observe their performance and quality in conditions different from its origin. The research was carried out in plots located in the countryside and in natural weather conditions. The quinoa varieties for the experiment were: Amarilla Marangani, Illpa Inía, Inía Salcedo, Altiplano, Hualhuas, Rosada Junín, Huancayo, INÍA 433, Pasankalla, Negra Collana, Amarilla Sacaca and Blanca Junín. Agroclimatic conditions, as well as soil and water were favorable for the production of quinoa. The results obtained were: The INIA 433 variety was the highest yield of 4.72 tons per hectare and $14.3 \%$ protein. It is a large and uniform panicle plant,
\end{abstract}

(C) Los autores. Este artículo es publicado por la Revista Campus de la Facultad de Ingeniería y Arquitectura de la Universidad de San Martín de Porres. Este artículo se distribuye en los términos de la Licencia Creative Commons Atribución No-comercial - Compartir-Igual 4.0 Internacional (https://creativecommons.org/licenses/ CC-BY), que permite el uso no comercial, distribución y reproducción en cualquier medio siempre que la obra original sea debidamente citada. Para uso comercial contactar a: revistacampus@usmp.pe. 
homogeneity of plants in growth and development was also observed. The variety of highest protein was the Yellow Marangani, with 16\% protein. The early quinoa varieties were: Illpa Inia, Inia Salcedo, Altiplano and Pasankalla (150-160 days of vegetative period)

Key words: Ayacucho, quinoa, varieties, quality, yield

\section{Introducción}

El Perú es un país con grandes potencialidades para el desarrollo agroexportador en quinua a través de instituciones como Sierra Exportadora, Agroideas y Procompite. La dirección agraria de los gobiernos regionales, provinciales y locales de Puno, Ayacucho, Huancavelica, Cuzco y Apurímac promueve su cultivo con la entrega de semillas, materiales y equipos. El aporte de productos y derivados alimenticios que la zona andina del país ha legado a la humanidad es de gran importancia como es el caso de la papa, olluco, oca, maca, yacón, quinua, kiwicha, cañihua, lúcuma entre otros (Romero, 2015 p.48).

En ese contexto, se han desarrollado diversos trabajos de investigación de variedades oriundas de esos lugares que fueron adaptadas a determinadas zonas ecológicas en búsqueda de alta productividad y calidad de la quinua, y así darle sostenibilidad a su participación en un mercado internacional. La quinua posee alto valor nutricional, adaptabilidad a diferentes condiciones agroecológicas (plasticidad genética), tolerancia a suelos salinos, resistencia a temperaturas extremas y aguante a la poca disponibilidad de agua. Adicionalmente, la quinua es un cultivo importante en la lucha contra el hambre a nivel mundial (IICA, 2015 p.15). En el año 2013, se sembró aproximadamente 132,000 hectáreas, por un valor de 52 millones de dólares. En estos últimos años, el crecimiento de la producción de quinua en los países de Bolivia, Perú y Ecuador representa el $80 \%$ de la producción mundial (FAO, 2013, p.1)

El mercado internacional requiere no solo de volúmenes sino de tamaño y calidad uniforme del grano a mejorar. Este proceso implica una adecuada normalización y estandarización. (Estrada, 2013.p.8).

La quinua tiene una extraordinaria adaptabilidad a diferentes pisos ecológicos. Soporta temperatura desde $-4^{\circ} \mathrm{C}$ hasta $38^{\circ} \mathrm{C}$. Es una planta eficiente en agua, es tolerante y resistente a la falta de humedad en el suelo. (FAO, 2011.p. 1).

Según Antelo (2010), la quinua se adapta a diferentes tipos de suelos y climas. Soporta muy bien la escasez de agua $y$ es considerada por la Organización Mundial de la Salud como uno de los alimentos recomendados para el futuro. (p.2)

Sierra exportadora (2013), recomienda enfocarse en la investigación genética y agronómica de la especie para la obtención de nuevas variedades resistentes y de alto rendimiento; en elevar la producción y su competitividad a fin de atender la creciente demanda mundial.

En 2013, la producción nacional de quinua alcanzó 52,124 toneladas. El primer lugar, lo ocupó la región Puno con 29,331 ton. El segundo lugar fue para Arequipa con 5,326 ton y el tercer 
lugar, Ayacucho con 4,925 ton. (Fairlie, 2015. p.13). También se encuentran áreas de cultivo como las existentes en el departamento de Ayacucho con condiciones adecuadas para su cultivo, que no están siendo aprovechadas (Chacchi, 2009. p. 2)

Sierra exportadora (2016) indica que la quinua es un alimento rico ya que posee los 10 aminoácidos esenciales para el ser humano. Esto hace que sea un alimento muy completo y de fácil digestión (p. 18).

La diversificación de productos elaborados de quinua depende en gran medida al conocimiento que se dispone sobre sus principales componentes químicos nutricionales, propiedades funcionales de los ecotipos para orientar su aplicación y uso agroindustrial (Reynaga, 2013).

Debido a su alto valor nutricional, adaptabilidad a diferentes condiciones agroecológicas (plasticidad genética) tolerancia a suelos salinos, resistencia a temperaturas extremas y a la poca disponibilidad de agua, la quinua es un cultivo importante en la lucha contra el hambre a nivel mundial (IICA, 2015, p. 15).

El departamento de Puno es el de mayor concentración de producción de quinua con cerca de 36,158 toneladas, seguido del departamento de Arequipa con 33,137 toneladas, Ayacucho, con 10,323 toneladas y Junín con 10,528 toneladas. (IICA, 2015, p.21). Por otro lado, en la provincia de Huamanga, perteneciente al departamento de Ayacucho, son productores de quinua los siguientes distritos: El Tambillo, Acocro, Vilcashuaman, Pampacangallo y
Accos Vinchos. También se ubica como productor el distrito de Chiara del centro poblado San Antonio de Manallasacc donde se ubican terrenos muy fértiles en óptimas condiciones agroclimáticas apropiadas para el cultivo de la quinua. En este lugar, se realizó el estudio de investigación. El objetivo fue señalar y describir cómo se realizó la adaptación de las variedades de quinua y determinar su rendimiento y calidad en las condiciones climáticas de C.C San Antonio de Manallasacc. Según Estrada (2013) en su estudio de la quinua en la estación experimental los Andenes (Cusco) muestra rendimiento con las variedades Negra Collana de $2.5 \mathrm{tm} / \mathrm{ha}$, Inía 415 Pasankalla de $3.5 \mathrm{tm} / \mathrm{ha}$, Inía 427 de $2.3 \mathrm{tm} / \mathrm{ha}$, la Rosada Junín de $2.5 \mathrm{tm} /$ ha, Blanca Junín de $2.5 \mathrm{tm} /$ ha y Amarilla Marangani de $3 \mathrm{tm} / \mathrm{h}$.

\section{Método}

\section{Indicadores considerados}

Agronómicos: rendimiento $\mathrm{Kg} / \mathrm{ha}$. $\mathrm{y}$ altura de planta $(\mathrm{cm})$

Calidad: nivel de proteína (\%) y granulometría (\% de granos de $2 \mathrm{~mm}$. $1.7 \mathrm{~mm} .1 .4 \mathrm{~mm}$ y de fondo)

Económico: rentabilidad (\%)

Se aplicó en el campo experimental en el fundo Pueblo Viejo de la comunidad campesina de San Antonio de Manallasacc en un área de $600 \mathrm{~m}^{2}$. En la unidad experimental se sembró tres surcos por variedad, cuyo largo fue de $33 \mathrm{~m}$ y $0.60 \mathrm{~m}$ el ancho en dirección de noroeste a sureste y $20 \mathrm{~cm}$ de distancia entre plantas.

La población es la totalidad existente de variedades de quinua a nivel 
nacional: 36 variedades comerciales con distintas y diferentes características de comportamiento en cada zona geográfica, en rendimiento y calidad del grano de la misma. La muestra tomada fue de 11 variedades de quinua. Se determinó de acuerdo a sus características edafológicas, agroclimáticas, altitud, agua y sanidad. Se usó de testigo a la Blanca Junín, la variedad que se siembra comúnmente en Ayacucho. Las semillas son variedades certificadas obtenidas de la estación experimental del Cuzco, Puno, La Molina, Huancayo y Ayacucho. Las diversidades de quinua para la presente investigación fueron:

- Blanca Junín. Es una variedad propia de la región central del Perú. Se cultiva intensamente en la zona del valle de Mantaro. También ha sido introducida con éxito en Antapampa, Cusco. Esta diversidad es resistente al Mildiu. Es de grano blanco y de tamaño mediano. Su periodo vegetativo es de 180 a 200 días. Su rendimiento es de $2500 \mathrm{~kg} / \mathrm{ha}$. Es la variedad testigo.

- Rosada Junín. Es una variedad de la región Junín desarrollada por la estación experimental Santa Ana- Huancayo del departamento Junín. Su tamaño de $1.2 \mathrm{~m}$. Los granos son dulces de color blanco con epispermo rosado. Su ciclo vegetativo es de 160 a 200 días. Su rendimiento de $2500 \mathrm{~kg} / \mathrm{ha}$.

- INIA 415 Pasankalla. Es una diversidad originaria de Puno. Se adapta entre los 3800 a 3900 metros sobre nivel de mar y soporta clima frío seco. Es una variedad óptima para la agroindustria y con rendimiento de $4500 \mathrm{~kg} / \mathrm{ha}$. Buena calidad de grano.

- Salcedo INIA. Es una variedad originaria de Puno y obtenida del cruce de las variedades Real Boliviana con Sajama. Posee grano grande (2.0 $\mathrm{mm}$ de diámetro), dulce, precocidad de 150 días de periodo vegetativo. Es tolerante a las heladas, sequías y al hongo común Mildiu. Se adapta a condiciones del altiplano, valles interandinos y costa, entre los 1284 a 3950 metros sobre nivel de mar. Su rendimiento comercial es de $2.5 \mathrm{Tm} /$ ha y su rendimiento potencial es de 4.0 Tm/ha.

- Hualhuas. Es una variedad de origen peruano boliviano. Su centro de producción es el departamento de Junín en el valle del Mantaro. Su periodo vegetativo es precoz, de 150 a 160 días. Tiene un potencial en rendimiento de 3,700 kg/ha. Grano de color blanco, de tamaño de 1.9 a $2.2 \mathrm{~mm}$, de sabor dulce y resistente al desgrane, Mildiu y Phoma. La variedad Hualhuas cuenta con una ventaja comparativa para el mercado norteamericano ya que posee un tamańo de grano adecuado para la exportación. Además, se trata de una variedad dulce, con un proceso fácil y a bajo costo se puede extraer el contenido de saponinas.

- INIA 433-Santa Ana/AIQ/FAO. Es una variedad originaria de Puno. Proviene del cruce de Sajama con Illpa INIA. Es de un periodo vegetativo de 160 días y un tamaño de grano grande, de color blanco cremoso, de adaptación desde 2800 a 3700 metros sobre nivel de mar con rendimiento de $3500 \mathrm{~kg} / \mathrm{ha}$ (potencial) y $2000 \mathrm{~kg} /$ ha (en campo). Es moderadamente tolerante al Mildiu.

- Illpa INIA. Es una variedad originaria de Puno. Resulta del cruce de Sajama y Blanca de Juli. 
Su periodo vegetativo es de 145 días, posee un grano de tamańo grande de 2 $\mathrm{mm}$. de diámetro, es de color blanco, dulce. $\mathrm{Su}$ rendimiento es de 3100 $\mathrm{kg} / \mathrm{ha}$. Es tolerante al Mildiu y a las heladas.

- Huancayo. Es una variedad proveniente del INIA Santa Ana - Huancayo de origen peruano boliviano. Su periodo vegetativo es precoz de 150 a 160 días, rendimiento potencial de 3,500 kg/ha. Grano de color blanco rosado, de tamańo de 1.8 a $2.1 \mathrm{~mm}$ de diámetro, de sabor dulce. Es resistente a Mildiu y Phoma.

- INIA 420 - Negra Collana. Esta variedad es de amplia base genética ya que es un compuesto formado por 13 accesiones provenientes de 12 localidades, comúnmente conocidas como "quytujiwras". Comercialmente se le asigna el nombre de INIA 420- Negra Collana y es el resultado de pruebas de identificación, adaptación y eficiencia desarrolladas participativamente con productores de las comunidades campesinas: Collana, Ccollpa, Cieneguilla, Vizcachani, Ilave, Mañazo y Pilcuyo de la Región Puno. Su mejor desarrollo se logró en la zona agroecológica Suni del altiplano entre los 3815 a 3900 ms.n.m., precipitación de 400 a $550 \mathrm{~mm}$ y temperatura de $4^{\circ} \mathrm{C}$ a $15^{\circ} \mathrm{C}$. Posee un grano de 1.6 $\mathrm{mm}$ de diámetro y de rendimiento de 3000 kilos porhectárea.

- INIA 431 Altiplano. Esta variedad fue desarrollada en la estación experimental de Illpa, de la región Puno. Se desarrolla desde el nivel del mar a 3700 metros sobre nivel de mar $\mathrm{Su}$ grano es blanco, de tamaño mediano, dulce y de periodo vegetativo precoz de 160 días. Su rendimiento es de $3,000 \mathrm{~kg} / \mathrm{ha}$.
- Amarilla Marangani. Es una variedad de origen peruano de la región de Puno, de periodo vegetativo tardío de 180 a 200 días. Se desarrolla de 800 a 3500 metros sobre el nivel de mar. $\mathrm{Su}$ potencial productivo es de 2500 $\mathrm{kg} / \mathrm{ha}$. Grano de color amarillo y de tamaño de 2.0 a $2.2 \mathrm{~mm}$ de diámetro, de sabor semidulce a amargo. Es resistente a Mildiu y Tumbado.

- INIA 427 Amarilla Sacaca. La quinua INIA 427- Amarilla Sacaca corresponde a la colección SP-AMSacaca procedente de la comunidad campesina de Sacaca-Pisac-CalcaCusco que en los procesos de evaluación y selección de las colecciones de las regiones Cusco y Apurímac demostraron ventajas competitivas. Tienen una adaptación óptima en los pisos de valles interandinos de las mencionadas regiones entre los 2750 hasta los $3650 \mathrm{~m}$ de altitud. Posee diámetro de grano de 1.6 a $2.2 \mathrm{~mm}$. y un rendimiento comercial de $2.3 \mathrm{tm}$ por hectárea.

La investigación se realizó en el centro poblado de San Antonio de Manallasacc del distrito de Chiara, fundo Pueblo Viejo, de propiedad del señor Juan Gómez, presidente de la Comunidad Campesina San Antonio de Manallasacc.

El clima es frío seco. Las lluvias varían de 400 a $1000 \mathrm{~mm}$. La temperatura media anual fluctúa entre 6 y $12{ }^{\circ} \mathrm{C}$. Las heladas son frecuentes por estas zonas; sin embargo, las temperaturas diurnas pueden llegar hasta $30^{\circ} \mathrm{C}$. La temperatura promedio anual es de $10^{\circ} \mathrm{C}$. Los datos climatológicos provienen de la estación meteorológica del proyecto Especial Río Cachi (PERC) en el distrito Los Morochucos ubicado a 3490 metros 
sobre nivel de mar. Es el más cercano a la zona de experimentación. El campo experimental pertenece a suelos de laderas, con relieve topográfico con pendiente que varía de 3 a $5 \%$. Se tomaron las mediciones y recolectó la muestra del surco del medio de 10 plantas tomadas cada tres metros al azar. Se realizó una encuesta y entrevista personal a los agricultores del lugar y pobladores del centro poblado.

\section{Resultados}

Tabla 1

Comparativo de características físicas y proteicas de variedades de quinua en Ayacucho

\begin{tabular}{|c|c|c|c|c|c|c|c|c|c|c|c|c|c|}
\hline \multirow[b]{2}{*}{ VARIEDAD } & \multirow[b]{2}{*}{ ORIGEN } & \multirow{2}{*}{$\begin{array}{c}\text { CICLO } \\
\text { VEGETA- } \\
\text { TIVO } \\
\text { (Días) }\end{array}$} & \multirow[b]{2}{*}{$\begin{array}{l}\text { TIPO DE } \\
\text { PANOJA }\end{array}$} & \multirow[b]{2}{*}{$\begin{array}{l}\text { LONGI- } \\
\text { TUD DE } \\
\text { PANOJA }\end{array}$} & \multirow{2}{*}{$\begin{array}{c}\text { PESO } \\
\text { GRANO } \\
\text { POR } \\
\text { PANOJA } \\
\text { (gr) }\end{array}$} & \multirow{2}{*}{$\begin{array}{c}\text { TAMAŃO } \\
\text { DE } \\
\text { PLANTA } \\
(\mathrm{cm})\end{array}$} & \multirow{2}{*}{$\begin{array}{c}\text { REN- } \\
\text { DIMIEN- } \\
\text { TO } \\
\text { (Tm/ha) }\end{array}$} & \multicolumn{6}{|c|}{ GRANO } \\
\hline & & & & & & & & COLOR & $\begin{array}{c}\text { PESO } \\
1000 \\
\text { GRANOS }\end{array}$ & $\begin{array}{c}\text { TAMAŃO } \\
(\mathrm{mm})\end{array}$ & SABOR & $\begin{array}{l}\text { RESIS- } \\
\text { TENCIA }\end{array}$ & $\begin{array}{c}\text { PRO- } \\
\text { TEINA } \\
\%\end{array}$ \\
\hline $\begin{array}{l}\text { AMARILLA } \\
\text { MARANGANI }\end{array}$ & Cusco & 210 & glomerulada & 57 & 92 & 147.7 & 3.7 & amarillo & 3.7 & 2 & Amargo & $\begin{array}{l}\text { mildiu, } \\
\text { granizada }\end{array}$ & 16 \\
\hline INIA SALCEDO & Puno & 160 & $\begin{array}{l}\text { amaranti- } \\
\text { forme }\end{array}$ & 42 & 46 & 128.8 & 1.87 & blanco & 3.2 & 1.8 & dulce & mildiu & 15.8 \\
\hline $\begin{array}{l}431 \text { INIA } \\
\text { ALTIPLANO }\end{array}$ & Puno & 150 & $\begin{array}{l}\text { amaranti- } \\
\text { forme }\end{array}$ & 47 & 36 & 128.6 & 2.6 & blanco & 3.3 & 2 & dulce & mildiu & 14 \\
\hline HUANCAYO & Junin & 220 & glomerulada & 47 & 40 & 152.8 & 3.25 & blanco & 3.6 & 1.8 & dulce & $\begin{array}{l}\text { mildiu, } \\
\text { granizada }\end{array}$ & 14.4 \\
\hline INIA 433 & Puno & 200 & glomerulada & 55 & 95 & 163.4 & 4.72 & blanco & 3.3 & 1.7 & dulce & mildiu & 14.3 \\
\hline $\begin{array}{l}\text { ROJA } \\
\text { PASANKALLA }\end{array}$ & Puno & 150 & glomerulada & 41 & 42 & 144.8 & 3.22 & rojo & 3.2 & 1.9 & $\begin{array}{c}\text { Semi } \\
\text { amarga }\end{array}$ & mildiu & 14 \\
\hline $\begin{array}{l}\text { INIA } 420 \text { NEGRA } \\
\text { COLLANA }\end{array}$ & Puno & 160 & glomerulada & 47 & 36 & 139.3 & 2.53 & negra & 3.1 & 1.6 & dulce & mildiu & 14.7 \\
\hline $\begin{array}{l}\text { AMARILLA } \\
\text { SACACA }\end{array}$ & Cusco & 230 & glomerulada & 47 & 87 & 135.5 & 3.17 & amarillo & 3.5 & 2 & $\begin{array}{c}\text { Semi } \\
\text { amarga }\end{array}$ & mildiu & 15.1 \\
\hline
\end{tabular}

Los resultados del experimento de la comparación de variedades de quinua en las condiciones agroclimáticas de la comunidad campesina San Antonio de Manallasacc durante los meses de noviembre a mayo en que se evaluó el rendimiento y calidad de la quinua orgánica para exportación tanto de las variedades precoces (160 días) y de noviembre a julio se evaluó las tardías (230 días) fueron los siguientes:

Las variedades precoces Illpa INIA, INIA Salcedo, 432 INIA Altiplano y la
Roja Pasankalla tuvieron rendimientos de 1.96, 1.87, 2.6 y $3.22 \mathrm{tm} / \mathrm{ha}$, respectivamente. El tipo de panoja de las tres primeras variedades es amarantiforme y la roja Pasankalla es glomerulada. En cuanto al tamaño de planta, las precoces varían en un rango entre 120 a 145 cm. El tipo INIA Salcedo tiene 15.8\% de proteína bajo estas condiciones. (AGQ PERU, 2015. P.3). Así mismo, las variedades tardías de Amarilla de Marangani, Hualhuas, Rosada Junín, Huancayo, INIA 433, INIA 420 Negra 
Collana, Amarilla Sacaca y Blanca Junín, tuvieron rendimientos de $3.7,4,15$, $3.21,3.25,4.72,2.53,3.17$ y 4.62 , respectivamente. El tipo de panoja de las variedades tardías es glomerulada. En cuanto al tamaño de planta está en el rango de 135.5 a $171.5 \mathrm{~cm}$. La variedad Amarilla Marangani tiene $16 \%$ de proteína bajo estas condiciones. (AGQ PERU, 2015. P.3)

\section{Discusión}

La mayoría de agricultores en Ayacucho selecciona semillas de la variedad Blanca Junín de sus cosechas anteriores $y$ no adquieren semillas certificadas $y$ desconocen variedades de corto periodo vegetativo, uniformidad del tamaño de panoja y tamaño de planta que permita mecanizar las cosechas.

Las cuatro variedades precoces encontradas en esta investigación son las de mayor perspectiva a desarrollar como cultivos promisorios para las tierras de la comunidad de San Antonio de Manallasacc. Cabe señalar, que se observó un mayor rendimiento y calidad en la variedad 431 INIA Altiplano en comparación a Illpa INIA, INIA salcedo y Roja Pasankalla, lo cual concuerda con la investigación realizada en Tarma (Rosas, 2015). Su maduración fisiológica es de 150 días de la variedad 431 INIA Altiplano concuerda con la investigación realizada en Puno (INIA, 2013, p.24)

El rendimiento de las variedades Blanca Junín e INIA 420 Negra Collana en el sector de Pumaranra, anexo de Kerapata, Abancay en Apurímac fue de 2.06Tn/ ha y $1.836 \mathrm{Tm} /$ ha respectivamente como señala (Cervantes 2016, p 71) lo cual evidencia rendimientos bajos en comparación con la producción de la comunidad campesina de Manallasac en Ayacucho.

Las variedades de quinua de panoja glomerulada son las de mayor rendimiento frente a las de panoja amarantiforme y la de mayor contenido proteico fue la Amarilla Marangani (16\%). La variedad INIA Salcedo es de corto periodo vegetativo y de mayor contenido proteico (15.8\%). La variedad de mayor rendimiento en el campo experimental a comparación de las demás variedades analizadas es INIA 433, de (4.72 tm/ha) que es una variedad tardía $\mathrm{y}$ tiene una mayor altura de planta.

\section{Conclusiones}

En conclusión, la producción de quinua en las zonas altoandinas de Ayacucho, a escalas industriales con manejo de alta tecnología y mecanizada es ideal para la exportación por el tamaño de grano blanco de $2 \mathrm{~mm}$. Por lo tanto, resulta recomendable la variedad 431 INIA Altiplano. Las variedades INIA 433 y la Blanca Junín son variedades de alto rendimiento por hectárea de $4.72 \mathrm{tm}$ y $4.62 \mathrm{tm}$ respectivamente en comparación de su producción en centro de orígenes de $2.0 \mathrm{tm} /$ ha y 2.5 $\mathrm{tm} /$ ha respectivamente(INIA,2013, p. y de $1.5 \mathrm{tm} /$ ha de la blanca Junín en Ayacucho (entrevista agricultores), pero baja en porcentaje proteico y de granos menor a $2 \mathrm{~mm}$, mientras que la Amarilla Marangani es la de más alto contenido proteico de $16 \%$ entre las variedades estudiadas. 


\section{Referencias}

AGQ PERU (2015). Informe de Ensayo. Labs \& Technological Services. 3 pág. Antelo, E. (2010). La Quinua: Grano de alta calidad y de gran demanda a nivel

Mundial. Comercio Exterior año 19N¹83 19pag. Junio 2010. Santa Cruz de La sierra-Bolivia.

Cervantes, N. (2016). Evaluación del rendimiento del cultivo de Quinua (Chenopodium quinoa Wildenow) en el sector Pumaranra, anexo Kerapata, del distrito de Tamburco. Tesis para optar el título de Ingeniero Agrónomo de la Universidad Tecnológica de los Andes, Abancay Apurímac 2016. 87 pág.

Chacchi, T. K. (2009). Demanda de la quinua (Chenopodium quinoa Wildenow) a nivel industrial. Tesis de postgrado de agronegocios de la Universidad Nacional Agraria La Molina. 2009. 144 pág.

El Peruano (2016). El grano de oro para el mundo. Fecha 09 de febrero 2016. Sección opinión.

Estrada, R. (2013). Cultivo de la quinua (Chenopodium quinoa Wild). en la Región Cusco. Programa Nacional Innovación Agraria en cultivos andinos de la Estación Experimental Agraria Los Andenes.

Fairlie, A. (2015). Cadena exportadora y políticas de gestión ambiental de la quinua en el Peru. Setiembre 2015. 64 pág.
FAO, (2011). La quinua: Cultivo milenario para contribuir a la seguridad alimentaria. Julio 2011

FAO-ALADI (2013). Tendencias y perspectivas del comercio internacional de la quinua. Diciembre 2012. 56 pág.

IICA, (2015). El mercado y la producción de quinua en el Perú. Lima: INIA IICA. 178 pág.

Programa de compensaciones para la competitividad (2014). Plan de negocios: Incremento del rendimiento y producción del cultivo de la quinua en la Asociación Agro productivo la Buena siembra del centro poblado de Manallasac, distrito de Chiara, provincia Huamanga en la Región de Ayacucho.2014. 64 pág.

Reynaga A., M Quispe, A Huarachi, I Calderón, J L Soto Quispe \& M Torrez (2013). Evaluación de las cualidades agroindustriales de los granos de quinua real. En Memorias del Congreso Científico de la quinua, La Paz, Bolivia, 14 y 15 de junio de 2013, 525-534.

Rosas, H., Geraldo, F. (2015). Evaluación agronómica de diez variedades de quinua (Chenopodium quínoa Wild), bajo dos sistemas de cultivo en la Unión- Leticia Tarma. Tesis para optar el título de ingeniero agrónomo de la Universidad Nacional Agraria La Molina, Lima. 
ESTUDIO COMPARATIVO EN RENDIMIENTO Y CALIDAD DE I 2 VARIEDADES DE QUINUA ORGÁNICA EN LA COMUNIDAD campesina de San Antonio de Manallasac, Ayacucho

Romero, V., Tirado, A., Dávalos, Sierra exportadora (2013).1ra Convención

J.Z. (2015). Determinación experimental mediante DSC de las estabilidades térmicas y las capacidades caloríficas: quinua, kiwicha y cañihua. Cátedra Villarreal, 3 (1) 47-52.

Internacional de quinua, de los andes al mercado mundial. 24 pág.

Sierra exportadora (2015). Perfil comercial de la quinua. Presidencia de Consejo de Ministro. Lima: (PCM). 
